A N N A L E S Annales de Bretagne et des Pays de l'Ouest

\title{
Un lycée dans la guerre. Le lycée de garçons de Rennes,
} 1939-1945

\section{Fabien Lostec}

\section{Q OpenEdition \\ 1 Journals}

\section{Édition électronique}

URL : https://journals.openedition.org/abpo/4308

DOI : $10.4000 / a b p o .4308$

ISBN : 978-2-7535-7720-6

ISSN : 2108-6443

\section{Éditeur}

Presses universitaires de Rennes

Édition imprimée

Date de publication : 18 décembre 2018

Pagination : 193-196

ISBN : 978-2-7535-7718-3

ISSN : 0399-0826

\section{Référence électronique}

Fabien Lostec, «Un lycée dans la guerre. Le lycée de garçons de Rennes, 1939-1945 », Annales de Bretagne et des Pays de l'Ouest [En ligne], 125-4 | 2018, mis en ligne le 18 décembre 2018, consulté le 28 février 2022. URL : http://journals.openedition.org/abpo/4308; DOI : https://doi.org/10.4000/abpo.4308 
tableau de l'histoire et de l'organisation de cet enseignement en France depuis 1959. Des renvois d'article à article auraient ici été utiles. Relevons les articles Défense scolaire, Caractère propre, Financement, Loi scolaire, Coût des études, Conseil régional, Immobilier, Organisation de l'enseignement catholique, Professeurs, Recrutement des personnel, etc. Une autre série (souvent sous la plume d'Yvon Tranvouez) aborde les déclinaisons de l'adjectif " catholique " accolé aux établissements. Là encore les enjeux abordés sont nationaux, et parfois même mondiaux, dans les articles Religion, Signes religieux, Aumônerie, Doctrine scolaire de l'Église, Religieuses, Vocations, Chrétiens contre l'école libre.

Terminons en soulignant quelques articles qui retiennent l'attention : les articles Jeunes filles et Mixité, les articles très originaux: Photo de classe, Parcs et jardins, Santé, Surnoms. L'article Mille-neuf-cent-quatre-vingt-quatre étonne (qu'est-ce donc que cette date?), mais éclaire le lecteur " extérieur " sur la sensibilité de l'enseignement catholique. L'historien appréciera les articles rétrospectifs Collèges d'Ancien Régime, ou Zouaves pontificaux, et les articles Établissements disparus, Juvénats, Petit séminaire (ici un renvoi aux articles sur chaque petit séminaire eut été utile). Si les personnels non enseignants actuels sont évoqués (Personnel Ogec), leurs prédécesseurs restent mal connus (quelques lignes impressionnantes dans l'article Religieuses). Même situation pour les professeurs : les prêtres diocésains professeurs ont peu attiré l'attention des historiens. Quelques articles sont trop courts : Bienfaiteurs et Entreprises (lien avec les) auraient gagné à être fusionnés. Un article manquant : "Ouest-France ", tant est fort le soutien de ce quotidien régional à l'enseignement catholique.

Un regret : l'ouvrage utilise le découpage régional de 1964, toujours en place. Certains seront chagrinés de l'exclusion de la Loire-Atlantique. D'autres estimeront que le cadre significatif aurait été un Grand Ouest intégrant tous les départements où l'enseignement catholique tient une forte place dans le second cycle (l'ancienne académie de Rennes plus les départements de la Manche et de l'Orne). Mais la Région Bretagne tenait sans doute à rappeler qu'elle a la charge des lycées. Un ouvrage qu'il ne faudrait pas réserver aux bibliothèques des lycées catholiques.

Jean-Luc MARAIS

BuRGuin, Pascal, Un lycée dans la guerre. Le lycée de garçons de Rennes, 1939-1945, préface d'Alain-François Lesacher, Rennes, Association pour la mémoire du lycée et du collège de Rennes (AmELYCOR)/Société archéologique et historique d'Illeet-Vilaine, 2017, 168 p.

L'ouvrage de Pascal Burguin se situe à la croisée de la recherche historique et de la démarche pédagogique, pour ne pas dire civique. À son origine, il y a un travail effectué avec différentes classes de Première du lycée Émile Zola dans le cadre du concours national de la Résistance et de la Déportation (CNRD) de 2009 à 2012 (le lycée de garçons de Rennes prend le nom de lycée Chateaubriand en 1960 puis de lycée Émile-Zola en 1971). Travail complété par les recherches de l'auteur aux Archives départementales d'Ille-et-Vilaine et aux Archives municipales de Rennes, ainsi que par les documents rassemblés depuis plusieurs années par l'AMELYCOR. Disons-le d'emblée, son aboutissement sous forme de livre est une réussite. Agrémenté de nombreuses illustrations, ce dernier nous renseigne sur la vie quotidienne d'un lycée durant la Seconde Guerre mondiale. Partant, il fait progresser la connaissance d'un sujet quelque peu délaissé par les chercheurs. En effet, alors que l'école primaire sous l'Occupation a récemment été analysée par Matthieu 
Devigne, aucune étude d'ensemble ne s'est intéressée aux lycées (DEvigne, Matthieu, L'école des années noires. Une histoire du primaire en temps de guerre, Paris, Presses universitaires de France, 2018) mais le lycée de garçons de Rennes abrite également un " petit lycée ", c'est-à-dire les classes du primaire. De même, les travaux sur un établissement scolaire en particulier, qui ne se limitent pas à une partie de sa vie ou à la seule question de la Résistance, sont rares. À titre d'exemple, on peut se référer à l'étude consacrée au lycée briochin Anatole Le Braz, De la nuit à l'aurore. Des lycées dans la guerre 1939-1945, Saint-Brieuc, Lycée Anatole Le Braz, 1995; ou encore à l'article d'Yves RANNOU, "Établissements scolaires et enseignement à Rennes pendant la Seconde Guerre mondiale ", Bulletin et mémoires de la Société archéologique et historique d'Ille-et-Vilaine, tome cx, 2006, p. 331-354.

Le livre se divise en trois grandes parties, toutes marquées par la volonté de l'auteur d'inscrire son propos entre trajectoires individuelles et portrait de groupe. La première est dédiée au fonctionnement et à l'adaptation du lycée au contexte de guerre et d'occupation. La seconde s'intéresse à l'engagement des élèves et des enseignants, que ce soit dans les rangs de la Résistance ou de la Collaboration. Quant à la dernière partie, elle fait un sort au souvenir et à la construction d'une mémoire lycéenne de la guerre. L'ensemble est complété par le témoignage inédit de Samy Mizrahi, lycéen rennais déporté à Auschwitz en 1944.

À la veille du conflit, le lycée de garçons est l'un des deux établissements publics d'enseignement secondaire à Rennes, avec le lycée de filles fondé en 1906. Il est le seul lycée de garçons du département et accueille " tous les niveaux d'étude depuis les classes élémentaires du "petit lycée" jusqu'aux classes préparatoires aux grandes écoles ", les seules dans lesquelles on trouve également des filles (p. 5). Dès 1939, sa vie est perturbée : alors que la culture de guerre s'immisce dans ses classes, plus de la moitié de son personnel est mobilisée et, comme en 1914, une partie de ses bâtiments sont réquisitionnés pour y installer un hôpital militaire. Dans des locaux devenus trop petits, le lycée doit de surcroît gérer un effectif en surnombre du fait de l'accueil d'élèves de l'est de la France et de la région parisienne poussés par le souffle de l'évacuation ainsi que des jeunes vivant dans des villes exposées au risque de bombardements allemands. Un premier brassage de populations s'effectue alors derrière les pupitres, prélude à celui de mai 1940, provoqué par l'exode qui fait suite à l'offensive allemande. Les élèves des classes préparatoires parisiennes ayant intégré le lycée participent à d'" heureuses répercussions sur les résultats de l'établissement aux concours des grandes écoles " (p. 15).

Pendant les mois de mai et juin 1940, les désagréments sont d'une autre nature. Des enseignants et des lycéens sont mobilisés ou s'engagent volontairement dans l'armée et les cours sont suspendus, ce qui oblige à décaler les vacances pour rattraper une partie des jours perdus. Parallèlement, le lycée devient un refuge pour les familles rennaises frappées par les attaques de l'aviation allemande. À la rentrée de 1940, les effectifs du lycée retrouvent leur niveau habituel mais l'absence de quelques professeurs oblige le proviseur à faire appel à des suppléants et à des enseignantes, dans un établissement dont le corps professoral était jusqu'ici exclusivement composé d'hommes. Le lycée est alors aux deux tiers occupé par les Allemands, qui y installent des services de la Feldpost et de la Luftwaffe : il devient donc un lieu important, qui est progressivement "bunkerisé " (p. 57). Un certain nombre d'élèves refusent de s'accommoder de cette cohabitation forcée. Les incidents entre les occupants et les lycéens se multiplient, obligeant à la construction d'une séparation au cœur de l'établissement. Plus largement, l'occupation du lycée provoque un réaménagement de l'espace, des rythmes et des activités scolaires. En 1943, Rennes étant la cible des bombardements alliés, les écoles sont fermées et les cours une nouvelle fois suspendus. Les élèves sont évacués dans diverses 
communes du département, ce qui provoque une déperdition des effectifs et une recrudescence des cours clandestins. Durant plusieurs mois, en 1943-1944, le lycée fonctionne " aux champs" (p. 61). Les élèves sont dépaysés : d'un côté ils disposent d'une plus grande liberté et font l'apprentissage de l'autonomie, de l'autre ils doivent faire face à l'éloignement parental et à des conditions de vie spartiates. Cet exil est parfois synonyme d'affrontements entre les élèves issus de la bourgeoisie rennaise et les jeunes ruraux, de milieux souvent plus populaires. Avec le débarquement du 6 juin 1944 s'ouvre une dernière séquence marquée par les bombardements et les dynamitages allemands qui touchent gravement l'établissement. Les travaux de reconstruction ne prendront fin qu'en 1955, onze ans après la Libération.

Durant l'Occupation, le lycée doit s'adapter à la nouvelle politique scolaire impulsée par Vichy. L'éducation est en effet refondée sur des bases " nouvelles » telles que la discipline, la morale, le respect de la hiérarchie ou encore le retour à la tradition (p. 24). Souhaitant disposer d'un corps professoral obéissant et sûr, l'État français renforce son contrôle sur le personnel et n'hésite pas à épurer les fonctionnaires " indésirables" (francs-maçons, gaullistes et communistes notamment). Trois élèves juifs sont également exclus du lycée et déportés à Auschwitz en 1942-1943. Seul Samy Mizrahi reviendra après avoir pu constater que, selon ses propres mots, " la survie est une loterie, un pur jeu de hasard " (p. 141). Le gouvernement fonde par ailleurs des structures destinées à encadrer et endoctriner les jeunes Français (Chantiers de la Jeunesse ou Service civique moral par exemple). Il cherche ainsi à faire des élèves des membres actifs de la communauté nationale et à leur apprendre le travail manuel, en équipe et en plein air. Mais ces organisations sont de fait court-circuitées par la politique de collaboration de leur promoteur. Les exigences allemandes en matière de réquisition de main-d'œuvre (instauration du STO en février 1943) contribuent à en faire des " coquilles vides". En complément, le régime de Vichy intègre massivement les lycéens aux journées de solidarité nationale et multiplie les actions de propagande à l'intérieur des établissements. Culte de la personnalité du Maréchal, défense de l'empire colonial et promotion de l'Église catholique sont à l'ordre du jour (p. 33).

Suivant des rythmes désormais bien connus - grâce notamment aux travaux de Jacqueline Sainclivier sur la Résistance en Ille-et-Vilaine -, des membres du lycée commencent à résister dès 1940-1941, individuellement ou collectivement, de manière informelle, mais souvent en dehors de toute organisation durable. Pendant que d'autres font le choix de l'Angleterre et de la France Libre, ils inscrivent des slogans patriotiques sur les murs, arrachent les affiches de l'occupant, confectionnent des tracts et manifestent dans la rue lors des dates commémoratives. Insouciants, les lycéens représentent un public turbulent, " difficile à contenir et dont l'action pouvait faire tache d'huile ", ce que craignent les autorités, qui prennent dès lors des mesures coercitives (p. 84). Le rectorat les multiplie également avec pour principal objectif de conserver son autonomie de décision et ne pas se laisser imposer des sanctions plus lourdes de la part des autorités préfectorales. Mais toutes ces actions n'ont que peu d'effets sur le comportement des lycéens; une partie d'entre eux s'engagent même dans la Résistance organisée : celle des réseaux, des mouvements et des maquis, souvent dans leur région d'origine où ils trouvent de solides soutiens. Ils participent ainsi à l'augmentation des effectifs résistants de la fin de l'année 1943 au premier semestre 1944, dont une des principales causes demeure l'entrée en clandestinité des réfractaires au sто. Formant l'élite intellectuelle et sociale de la société, ils ne tardent pas à occuper dans la clandestinité des fonctions d'encadrement ou de formation. Même chose pour les enseignants qui se retrouvent parfois à leurs côtés, " créant ainsi une communauté inédite de destin entre maîtres et élèves " (p. 101). Communauté frappée par la répression et qui compte de nombreux fusillés ou déportés. Comme dans le reste de la société, les résistants forment une minorité des membres du lycée. Au-delà 
des rares pétainistes ou maréchalistes du lycée, plus opportunistes que convaincus, ils sont quatre fois moins nombreux à gagner les rangs des groupes collaborationnistes (RNP, groupe Collaboration et PNB notamment). Dans la Résistance comme dans la Collaboration, c'est l'effet de " groupe ", qui est le principal facteur explicatif de l'engagement des lycéens. Le rôle de la camaraderie, et notamment du "groupe classe ", ou celui de la famille détermine bien souvent les itinéraires empruntés par les élèves.

La Libération venue, l'ensemble des jeunes et des professeurs regagnent le lycée. Les personnes suspectées de collaboration sont inquiétées voire sanctionnées par l'épuration, non sans difficultés de la part des nouveaux pouvoirs pour " faire la part entre les fautes avérées et les règlements de compte partisans " (p. 123). Très vite vient ensuite le temps de la mémoire, ou plutôt des mémoires. Cette diversité, qui illustre le caractère total et la complexité du conflit, est parfaitement démontrée par l'auteur à travers l'étude de la plaque commémorative apposée dans le hall d'honneur du lycée, rassemblant, malgré quelques erreurs et omissions, l'identité des " morts pour la France ». L'apposition de cette plaque et les différentes cérémonies commémoratives permettent non seulement de rendre hommage aux victimes mais aussi de faire de l'établissement un lieu de mémoire, en créant une identité lycéenne collective voire commune. S'y côtoient les noms de soldats mobilisés en 1939-1940, de combattants volontaires de la France libre, de résistants torturés, fusillés ou déportés, d'otages exécutés, de victimes de bombardements mais aussi de Juifs morts en déportation (p. 128). C'est la spécificité du sort réservé à ces derniers que vient rappeler le témoignage de Samy Mizrahi. Il s'inscrit dans un paysage mémoriel qui opère aujourd'hui une distinction entre les différentes victimes du conflit, ce qui n'était pas le cas durant les années d'après-guerre, lorsque la priorité était de " préserver l'unité nationale brisée par la guerre " (p. 129).

Au terme de la lecture, quelques regrets peuvent cependant être émis. Tout d'abord en ce qui concerne le manque de références à l'historiographie de la période. Des études d'importance dont l'auteur s'inspire ne sont jamais citées. L'absence de bibliographie à la fin de l'ouvrage est de ce point de vue significative. Nous émettons ensuite une réserve au sujet des limites inférieures choisies pour définir la Résistance. Peut-on en effet ranger parmi la Résistance, fût-elle informelle et le fait d'adolescents, la recherche d'informations en écoutant la BBC (p. 75-76)? La transgression d'un interdit n'est pas nécessairement un acte de résistance. Enfin, davantage qu'une critique, nous nous permettrons d'évoquer une piste pour ce qui est de la répression des collaborateurs à la Libération. L'auteur l'a d'ailleurs peutêtre creusée mais il n'en dit mot. Il aurait en effet été intéressant de dépasser les archives des commissions d'épuration et de sonder celles des archives judiciaires afin de savoir si des élèves ou des enseignants furent jugés par la cour de justice ou la chambre civique d'Ille-et-Vilaine. Une démarche relativement aisée puisque les archives départementales conservent un cahier dans lequel sont recensés les noms de tous les justiciables de l'épuration des cinq départements bretons et qui aurait éventuellement permis d'accéder à des sources d'une très grande richesse. L'étude aurait ainsi pu aborder une question très intéressante et là encore délaissée par l'historiographie : celle des mineurs face à la justice de l'épuration.

Ces quelques remarques n'enlèvent rien de son intérêt et de sa richesse à cet ouvrage qui nous renseigne avec force détails et réflexions percutantes sur la vie d'un lycée qui, malgré toutes les difficultés auxquelles il est confronté durant la Seconde Guerre mondiale, a finalement démontré sa capacité à poursuivre sa mission d'enseignement, " en puisant dans ses ressources fonctionnelles mais aussi en s'adaptant constamment à la situation " (p. 9). 